\title{
A New Quantitative Quality Measure for Machine 'Iranslation Systems
}

\author{
Keh-Yih Su${ }^{\dagger}$, Ming-Wen $\mathrm{Wu}^{\ddagger}$ and Jing-Shin Chang ${ }^{\dagger}$ \\ tDepartment of Electrical Engineering \\ National Tsing-Hua University \\ Hsinchu, Taiwan 300, R.O.C. \\ email:kysu@ee.nthu.edu.tw, shin@ee.nthu.edu.tw
}

\author{
${ }^{\ddagger}$ Behavior Design Corporation \\ $2 \mathrm{~F}, 28, \mathrm{R} \& \mathrm{D}$ Road II \\ Science-based Industrial Park \\ Hsinchu, Taiwan 300, R.O.C.
}

\begin{abstract}
ABSTRAC'T
In this paper, an objective quantitative quality measure is proposed to cvaluate the performance of machitc translation systems. The proposed method is to compare the raw translation output of an MT system with the final revised version for the customers, and then compute the editing efforts required to convert the raw translation to the final version. In contrast to the other proposals, the evaluation process can lo done quickly and automatically. Hence, it can provide a quick response on any system change. A system designer can thus quickly lincl the advantages or faults of a particular performanceimproving strategy and improve system performance dynamically. Application of such a measure to improve the system performance on-line on a parameterized and fecdback-controlled system will be demonstrated. Furthermore, because the revised version is used directly as a reference, the perfonnance measure can reflect the real quality gap between the system performance and customer expectation. A system designer can thus concentrate on practically important topics rather than on theoretically interesting issues.
\end{abstract}

\section{Introduction}

There are several reasons performance measure is required while building machine translation systems. (1). Potential customers need to be able to compare the performance of different systems. (2). System designers would like to keep abreast of the current system performance, and make sure the system keeps on improving, not subject to the flip-flop problem. (The flip-flop problem is caused when system designers try to fix some problem of the system without a thorough test. While that problem is solved, other problems may pop up. This kind of problem becomes more serious when the system scales up.) (3). The measure feedback will highlight correct rescarch direction. (4). In a parameterized system, if a quantified cost function is provided, it can be used directly for parameter tuning. Thus, a systernatic and standardized approach for performance evaluation and the establishment of a common testing base are urgently required.

Most conventional approaches evaluate system performance by human inspection and subjective measures. While post-editing, the post-editors can provide feedback on the quality of machine translation, which then is used for dictionaty update and linguistic analyses of crrors [Ross 82]. Also, feedback can be obtained from professional translators who annotate carefully on the printouts of raw translation output [Pigo 82]. From human feedback, system designers tend to overtune the system. Another approach, proposed in [King 90], is to collect the test suites and divide them into two sections: one to look at source language coverage, the other to cxanine translational problems. Such an approach can avoid the over-tuning problem caused by human feedback. The advantage of human inspection is that humans can pinpoint the real linguistic problems and make corrections. However, there are several disadvantages: (1). It is tow costly for human inspection of the translation output quality. To get significant statistics on the real system performance, a large volume of text must be provided. The cost for human inspection is thus extremely high. (2). It will take too long for the results to come out. For this reason and the cost consideration, it can not be repeated frequently. Therefore, it can not provide a quick suggestion to a system designer when the system is changed or when the domain is alerted. For a system that must handle a wide variety of types of text, it fails to provide immediate help to adapt to the particular domain or field. (3). It is not easy to achieve consistency and objectiveness. Even for the same person, it is very likely that he/she would judge a translation result differently at different time, especially when the evaluation criteria are loosely defined.

Based on the above problems with human inspection, some automatic approaches were proposed to eval- 
uate translation output quality. In [Yu 91], for example, a corpus of 3,200 sentences were collected. Then, some test points are selected by linguists based on the sentences. in the corpus. The test points are what linguists think the most important features for the sentences in the corpus. Each test point is assigned a weight according to its imporlance in translation. The test points are coded in programs, therefore the testing can be done automatically. The advantage of this approach is that since their criteria are purcly linguistic, they can do a very delicate evaluation and find the real linguistics problems involved. However, to acquire significant statistics on the performance, a large corpus is required. Corpus collecting and test points selecting are very time-consuming. Furthermore, to achieve high grade in quality with respect to these test points, the system might be over-tuned to the set of particular test points such that they fail to reveal their real performance on a broader domain. The system designer might thus be misled by such a close-test or training-set performance and have an over-optimistically evaluated figure of performance. (Sec [Devi 82, Chapter 10] for detailed comments on performance evaluation.)

We propose a new quantitative quality measure to evaluate the performance of machine translation systems. The method is to compare the raw translation output of an MT system with the final revised version for the customers, and then the editing efforts required to convert the raw translation to the final version is computed. Compared with the above proposals, the evaluation process can be done quickly and automatically. Moreover, application of such a measure to improve the system performance on-line on a parameterized and feedback-controlled system is easy. Finally, since the revised version is used directly as a reference, the performance measure can reflect the real quality gap between the system performance and customer expectation.

\section{Performance Evaluation Using Bi-Text Corpus}

\subsection{Criteria for a Good Measure}

From the above discussion, it is desirable to have a performance measure and a performance evaluation process with the following properties:

[1] low cost: minimal human interference is involved and can be done automatically.

[2] high speed: it can give system designers quick response and immediate help (even on-line, for a parameterized system); it can also provide positive stimulation to the system designer psychologically

[3] exactness: the difference between customer expectation and real system performance can be reflected.
Because the design goal of a system is to optimize some gain or minimize some cost, a good performance measure is definitely an important factor on the improvement of the system.

\subsection{A Distance Measure Approach}

To achieve the goals outlined in the previous section, a quantitative measure is proposed. In our approach, we first establish a bi-text corpus composed of source language sentences and the corresponding target language sentences. The target sentences are the revised version of the raw translation which were post-edited to tailor to the customers' need (for publication). Therefore, the target sentences are what customers really want. Then, we employ a distance measure method to evaluate the minimum distance between the raw translation output and the target sentences in the bi-text corpus. By distance, we mean the editing efforts needed to edit the raw translation output into the revised version. In other words, we would like to know the number of key strokes required to bc performed for such editing. The smaller the distance is, the higher the translation output quality is.

The sentence pairs in the bi-text corpus is the source sentence and the target sentence post-edited for the customers. The reason for adopting the revised version text as the measure reference is that even the machine translated texts are error-free judged by system designers, it may not be the final version customers really want. In general, the system designers, who are aware of the limitation and restriction of an MT system, tend to give loose quality criteria, and thus an overoptimistic evaluation. Human inspection can only achieve correctness and readability, but the acceptability to customers is usually low. We try to offer customers the solution they really need. Thus, every trial to fine-tune the output quality should be directed to fit customers' needs [Chen 91, Wu 91].

This approach has several advantages over other methods: (1) Since the final revised version is used for comparison, it will reflect the real quality gap between the capability of the system and the expectation of the customers. According to our experience on providing translation services with the ArchTran MTS, for most translation materials, even for manuals or announcement, the final versions are intended for publication, not just for information retrieval. Therefore, traditional quality measures which are graded loosely like 'correct', 'understandable', ... and so on, provides little information on how the system should be tuned. Thus, it's reasonable to adopt the final revised version as the measure reference. (2) Human power is more expensive than computer power. Since this approach involves no human interference, the evaluation cost is fairly low. (3) The current system performance can be reported very soon because of high computer speed. With the quick feedback, more performance improving strategies can be tried out, and thus research efficiency is improved. (4) We can show improvement to raise research morale and excite enthusiasm, for a clear indicator of performance improvement 
is the strongest incentive for $R \& D$ engineers. System problems can be located and solved quickly, thus a lot of work is saved. (5) Because the final revised version is used as the measure reference, the text can be classified into different domains and styles. With the quick fecolback, it can help adapt system to different domains and styles.

\subsection{Distance Measure and Weight}

\section{Assignment}

Four primitive editing operations are defined, namely insertion, deletion, replacement and swap [Wagn 74, Lowr 75]. Since each operation requires different editing efforts, different weights must be assigned. We assign the weight according to the number of key strokes needed for each operation under the most popular editor. For Chinese editing, the weights we assign for insertion is 5, deletion 1, replacement 5 and swap 6. The deletion operation is the least costly operation for its simplicity. The insertion and replacement operations take more efforts including cursor addressing, entering and leaving editing mode. The swap operation needs a little more effort than insertion and deletion. (The swap cost is defined here to be the cost of one insertion plus one deletion. For a post-editing facility with a special swap editing function, the swap cost should be a function of the distance between the characters to be swapped. This cost might be less than the cost of one insertion plus one deletion for adjacent words. For the present, the cost is used for simplicity.)

\subsection{Alignment}

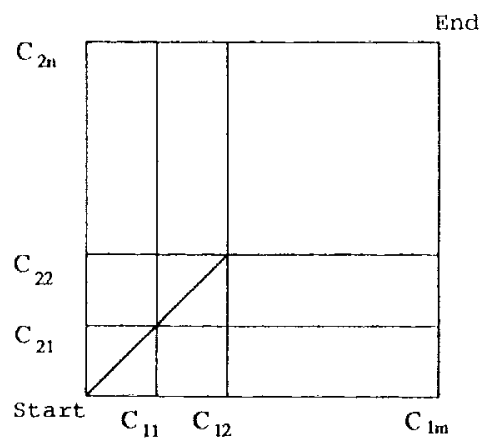

\section{Figure 1. Alignment of raw output sentence with revised version sentence}

The evaluation of the distance between the raw output sentence and the final revised sentence can be formulated as a "shortest path" searching problem. The problem can be solved with the well-known dynamic programming rechnique [Bell 57]. Figure l shows a diagram for the dynamic programming problem. Assume that $H=\left\{c_{11}, c_{12}, \ldots, c_{1 m}\right\}$ is the raw output senteice composed of $m$ characters $c_{11}$ through $c_{1 m}$, and $Q=$ $\left\{c_{21}, c_{22}, \ldots, c_{2 n}\right\}$ is the final version sentence composed of $n$ characters $c_{21}$ through $c_{2 n}$.

In Figure 1, the big square has $m \times n$ grids with weight (cost or distance) associated with each edge and diagonal. Many paths can be picked from the Stant to the End. Any path along the edge or diagonal from the Start to the End represents a sequence of editing operations that chariges the raw output sentence to the final revised sentence. The cost incurred for each path is the accumulative weights along the path travelled. The cost/distance of a path stands for the editing efforts to make the changes. 'Therefore, the minimum distance path stands for the least cost. The goal is to pick the path with the minimum cost, or shorkest distance.

There are three directions to go at each position: right, up or up-right. We can make an analogy between linding the shortest path and jerforming the fewest editing operations to convert the raw output sentence into the final version sentence. When we are at the Start point, we have the raw output sentence. If we go right, a deletion operation is performed. If we go upward, an insertion is performed. If we go along the diagonal, either one of two cases will happen. When $c_{1 i}$ and $c_{2 j}$ on the two edges of the diagonal are the same, no operation is performed, and no cost is incurred. If, however, they are different, a replacement is performed. When we eventually reach the End point, we have edited the raw output sentence into the final version sentence. The sccond path Iraversal is required to compute the number of swap operation. If deletion of one character is to be performed, and that character will have to be inserted in the following opcrations, then one delction and one insertion are replaced by onc swap operation. By the same token, If the inserted character will be deleted later, the insertion and deletion are saved by jerforming one swap. If the shortest path is picked, then we have edited the sentence with least effort.

The distance between the raw output sentence and the revised sentence can be formulated as follows:

$$
D \equiv w_{i} \times n_{i}+w_{d} \times n_{d}+w_{r} \times n_{r}+w_{s} \times n_{n}
$$

where $n_{i}, n_{d}, n_{r}$ and $n_{s}$ are the numbers of operation for insertion, deletion, replacement and swap performed respectively; $w_{i}, w_{d}, w_{\text {, and }} w_{s}$ are the weights for thesc operations. $D$ is the total distance for one specific editing sequence; that is to say, $D$ is the number of key strokes required to post-edit the raw translation sentence into the final version sentence.

\subsection{An Example}

The solid path in figure 2 gives an example to show the steps performed using dynamic programming to find the least cost for editing one sentence. The raw output sentence is "This is my own computer" in the $X$ axis, and the revised version sentence is "This 
computer is mine" in the $\mathrm{Y}$ axis. One insertion and deletion along the path are marked an " $X$ " because the word "computer" appears in different locations in two sentences. Therefore, a swap operation is performed to save one insertion and one deletion. Totally, there are one replacement ("my" to "mine"), one deletion ("own") and one swap ("computer"). Table 1 shows the path with the least cost. The first row in Table 1 is the raw output sentence, the second row the revised sentence, and the third the editing operations performed. The least cost is 12 (i.e., $1 \times 5+1 \times 1+1 \times 6=12$ ). And, the average cost is 2.4 per word. Note the difference between such a measure with a conventional subjective approach. The two sentences might be judged as equally readable and understandable by human inspection. However, to tailor to the final output for publication, we still need 2.4 units of cost per word. If we follow the dotted path in Figure 2 , we will get the path not of the least cost. Table 2 shows this path. In this case, there are one deletion and threc replacements. The cost incurred is 16 (i.e., $1 \times 1+3 \times 5=16$ ).

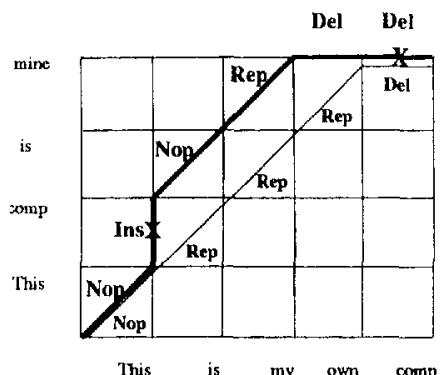

Ins : Insertion

Del : Deletion

Rep : Replacement

Nop : No Operation

Figure 2. An example to show the steps in dynamic programming

\begin{tabular}{|c||c|c|c|c|c|c|}
\hline Raw & This & & is & my & own & comp \\
\hline Rev & This & comp & is & mine & $X$ & \\
\hline $\begin{array}{c}\text { Edit } \\
\text { Op }\end{array}$ & NOP & SWAP & NOP & REP & DEL & \\
\hline
\end{tabular}

Table 1. A path of the least cost 12

\begin{tabular}{|c||c|c|c|c|c|}
\hline Raw & This & is & my & own & comp \\
\hline Rev & This & comp & is & mine & $X$ \\
\hline $\begin{array}{c}\text { Edit } \\
\text { Op }\end{array}$ & NOP & REP & REP & REP & DEL \\
\hline
\end{tabular}

Table 2. A path of cost 16

\section{Application to Performance Evaluation and Improvement}

As discussed above, the most direct application of the preference measure is, of course, to show the current status of the system performance. This function directly serves several purposes.

11] With this performance measure applied to a large bitext corpus, one can show to the potential customers the current system performance in terms of the editing efforts required to get high quality translation. Furthermore, because the performance measure is an objective measure, it can be used to compare the system performance with other systems based on the same testing base.

[2] The quick response makes it possible for the system designers and the linguists to get a clear idea about the advantages or faults of a particular strategy or formalism. From the quick feedback of the measurement, one can try different approaches in rather short time. Hence, the research pace will be accelerated rapidly. And the system designers can make sure the system is on the right track.

[3] Psychologically, a clear indicator of performance improvement is the strongest incentive for $\mathrm{R} \& \mathrm{D}$ teams. According to our working experience, the research team members tend to become upset when their ideas can not be fully implemented and justified in a reasonable lime. With a clear performance indicator and quick response, the team members usually get excited and their morale is raised substantially.

The following case study shows how the quick and automatic performance evaluation method help make decision on some designing issues and highlight research directions. In a recent evaluation run, a bi-text corpus, containing 6,110 English-Chinese sentence pairs are used to evaluate a particular version of the ArchTran EnglishChinese MT system. The Chinese sentences are the revised version of the corresponding English sentences, which are to be published as a Chinese technical manual. The revised Chinese sentences are used as the reference for comparison with the unrevised version. The editing effort required to post-edit the unrevised version is then evaluated using the proposed distance measure. It takes only about 30 seconds to get the required measure. The experimental results are shown in Table 3.

At first, we think the editing cost might be too high to get the required high quality, and we suspect that the probabilistic disambiguation mechanism for the analysis module [Chan 92a, Liu 90, Su 88, 89, 91b] might not be properly tuned. So we use an adaptive learning algorithm [Amar 67, Kata 90, Su 91a] to adjust the probabilistic disambiguation modules of the system. Table 4 shows the comparison of the original status of the MTS and its best-tuned case. In the best case, the translation with the least cost is selected. 


\begin{tabular}{|l|r|}
\hline total number of sentences & 6,110 \\
\hline total number of Chinese characters & 135,318 \\
\hline total number of insertions & 23,541 \\
\hline total number of deletions & 21,238 \\
\hline total number of replacements & 22,721 \\
\hline total number of swaps & 9,953 \\
\hline number of insertions per sentence & 3.85 \\
\hline number of deletions per sentence & 3.48 \\
\hline number of replacements per sentence & 3.72 \\
\hline number of swaps per sentence & 1.63 \\
\hline total cost for 6,110 sentences & 312,266 \\
\hline cost per sentence & 51.11 \\
\hline cost per claracter & 2.31 \\
\hline
\end{tabular}

Table 3. Statistics summary of distance measure (Editing cost scheme: Insertion 5, Deletion 1, Replacement 5 and swap 6)

\begin{tabular}{|l||c|c|}
\hline & Original status & Best case \\
\hline Total cost & 312,266 & 289,290 \\
\hline Cost per sentence & 51.11 & 47.35 \\
\hline Cost per word & 2.31 & 2.14 \\
\hline
\end{tabular}

Table 4. Performance of the original status and of the best case

Table 4 does show some improvement after tuning the disambiguation module. However, the improvement is not apparent. This implies that the disatnbiguation part is not the major bottleneck for the quality gap. In fact, most translations are readable and understandable under luman's judgement. So we examined the other parts of the system. We found that the biggest problem is that the translation style does not fit customers' need. We thus conclude that more efforts should be concentrated on the transfer and generation phases, and a transfer and generation model that is capable of adapting the system to different domains and styles [Chan $92 \mathrm{~b}$ ] is required. This case study shows that a quick performance evaluation does play an important role in directing the research direction.

\section{Parameterized Feedback Control System}

\section{Based on the Performance Measure}

Through the quick and automatic quantitative distance measure, the system performance can be on-line reported in terms of an objective cost function. Therefore, it can be applied in the guided searching in a parameterized, fecdback controlled system. The following sections show how the quick performance measure helps to construct such a feedback system. Without a quick performance evaluator, these models will not be made possible.

\subsection{Ambiguity Resolution and Lexicon Selection in a Feedback System}

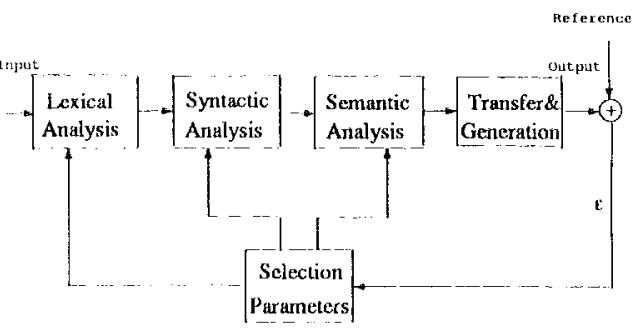

Figure 3. Parameter tuning from feedback

A parameterized feedback-controlled MT system can be modeled as in Figure 3. The control of the system is govened by its static knowledge and a dynamically adjustable parameter set which are used to select the best interpretation among the various ambiguities, or to select the most preferred style in the transfer and generation phases. The probabilistic translation model proposed in [Chen 91] is one such example. In this model, the best analysis is to be selected to maximize an analysis score or score function [Chan 92a, Liu 90, Su 88, 89, 91b] of the following form:

$$
S c o r c \equiv P\left(S e r_{1}, S y m_{j}, L e x_{k} \mid \text { Words }\right)
$$

where $S e m_{i}, S y n_{j}, L e x_{k}$ is a particular set of semantic annotation, syntactic structure and lexical catcgory corresponding to some ambiguous construct of the sentence $W$ ords. Furthermore, the best ransfer and generation is to be sclected to maximize the following transfer score $S_{t x}$ and generation score $S_{\text {ves }}$ :

$$
\begin{aligned}
& S_{t x f}=P\left(T_{i} \mid T_{0}\right) \approx P\left(\hat{T}_{i} \mid \hat{T}_{0}\right) \\
& S_{\text {gen }} \approx P\left(t \mid \hat{T}_{i}\right)
\end{aligned}
$$

where $T_{t}, T_{a}$ are the target and source of intermediate representations in the form of an annotated syntax tree (AST); $\hat{i}, i, i$, are the normalized version of the AST"s, called the normal forms (NF) of the AST's, which are 
used particularly for transfer and generation, and $t$ is the generated target sentencc [Chan 92b].

In such a system, we are to formulate a model as closely to the unknown real language model as possible. Thus, the main task is to estimate the parameters which characterize the model. Because it is not always possible to acquire sufficient data, particularly for complex language models, the estimated parameters might not be able to characterize the real model. Under such circumstances, we can adaptivcly adjust the estimated parameters according to the error feedback.

In Figure 3, the analysis phase (lexical, syntactic and scmantic analyses) of the system is characterized by a set of selection parameters. The input is fed into the lexical analysis phase. The output is generated and acts as the input of transfer phase. In the feedback controlled scheme, a set of revised text, such as the 6,110 Chinese sentences in the previous section, can be uscd as the reference, and be compared with the translation output of 6,110 sentences. Under the scoring mechanism, the preferred analysis selected may not correspond to the translation with the shortest distance from the reference. Under this circumstance, we can adjust the selection parameters according to the error $\epsilon$ (the difference between the reference and the system output) iteratively. Through this adaptive learning procedure [Amar 67, Chia 92, Kata 90), the estimated parameters will approach the real parameters very closely. In this way, it will help in ambiguity resolution and lexicon selection. Such a system is made possible to automatically fine-tune the system because the performance measure proposed in this paper provides an on-line response to the iteratively changed parameters.

\subsection{Bi-lingual Transfer Model}

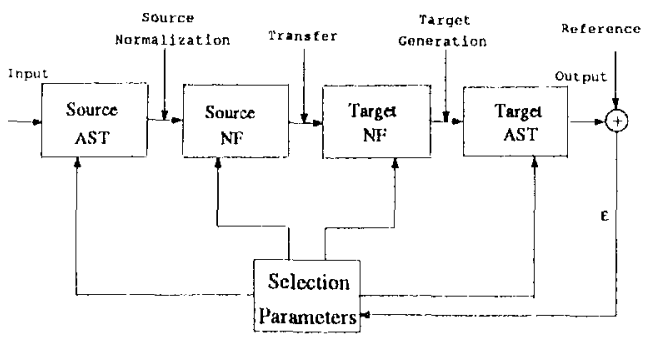

AST : Annoteted Syatix Tree

NF : Nontiel Form

Figure 4. An adaptive learning conceptual model for the transfer and generation phases

As another application of the quick performance measure, we can construct a fecdback controlled transfer and generation model. Figure 4 shows such a conceptual model for the parameterized transfer and generation phases [Chan 92b], where AST [Chan 92a] is a syntax tree whose nodes are annotated with syntactic and semantic features and NF is a normalized version of AST, which consists of only atomic transfer units. (See also the previous section for the transfer score and generation score). The Source NF and Target NF are characterized by a set of selection parameters. By jointly considering the parameters characterizing the Source and Target NF, we can adaptively adjust the parameters from the feedback of both directions just like in the previous section. Also the feedback control will make the parameters be tuned to fit the stylistic characteristics of the revised target sentences. Hence, more natural sentences could be generated and less editing effort could be expected in order to get high quality translation. Again, only a quick performance evaluator can make such feedback system practical.

\section{Conclusion}

The need for performance evaluation is rising, for both customers and system designers. We proposed a performance evaluation method with which system performance can be evaluated automatically and quickly. The approach to improve system performance and feedback controlled MT system is proposed based on such measure. Because the revised text is used directly as reference, the performance measure can indicate real quality gap between users' expectation and system capability.

Though we can not measure the very fine detailed features because there is not very much linguistic knowledge incorporated, our approach has many advantages over conventional approaches. There is no need for human interference. The criteria are consistent and objective. And, we are trying to offer the solutions what users really need. Most important of all, from the feedback of measurement, it is fairly easy for system fine-tuning.

\section{References}

[Amar 67] Amari S., 1967. "A Theory of Adaptive Pattern Classifiers," IEEE Trans. on Electronics Computers, vol. EC-16, pp. 299-307, June 1967.

[Bell 57] Bellman R.-E., "Dynamic Programming," Princeton University Press, 1957. Princeton, NJ, USA.

[Chan 92a] Chang, J.-S., Y.-F, Luo and K.-Y. Su, 1992. "GPSM: A Generalized Probabilistic Semantic Model for Ambiguity Resolution," to appear in Proceedings of ACL-92, 30th Annual Meeting of the Association for Computational Linguistics, University of Delaware, Newark, DE, USA, 28 June-2 July, 1992.

[Chan 92b] Chang, J.-S. and K.-Y. Su, 1992. "A CorpusBased Statistics-Oriented Transfer and Generation Model for Machine Translation," to submit.

[Chen 91] Chen, S.-C., J.-S. Chang, J.-N. Wang and K.Y. Su, 1991. "ArchTran: A Corpus-based Statistics- 
oriented English-Chinese Machine Translation System," Proceedings of Machine Translation Summit III, pp. 33-40, Washington D.C., USA, July 1-4, 1991.

[Chia 92] Chiang, T.-H., Y.-C. Lin and K.-Y. Su, "Syntactic Ambiguity Resolution Using A Discrimination and Robustness Oricnted Adaptive Leaming Algorithm," to appear in Proceedings of COLING92, 14th International Conference on Computational Linguistics, Nantes, France, 20-28 July, 1992.

[Devi 82] Devijver, P.A. and J. Kitticr, Pattern Recognition : A Statistical Approach, Prentice-Hall, London, 1982.

[Kata 90] Katagiri, S. and C.-H. Lee, 1990. "A Generalized Probabilistic Decent Mcthod," Proc. Acous. Sco. of Japan, pp. 141-142, Nagoya, Sep. 1990.

[King 90] King, M. and K. Falkedal, 1990. "Using Test Suites in Evaluation of Machinc Translation Systems," Proceedings of COLING-90, vol. 2, pp. 211-216, the 13th International Conference on Computational Linguistics, Helsinki, Finland, 20-25 Aug. 1991.

[Liu 90] Liu, C.-L., J.-S. Chang and K.-Y. Su, 1990. "The Semantic Score Approach to the Disambiguation of PP Attachment Problem," Proceedings of ROCLING-III, pp. 253-270, Taipci, ROC.

[Lowr 75] Lowrence, R. and R.-A. Wagner, 1975. "An Extension of the String-to-String Correction Problem,"Journal A.C.M., vol. 22, no. 2, pp. 177-83, 1975.

[Pigo 82] Pigoth, 1.-M., 1982. "The Importance of Fecdback from Translators in the Development of Highquality Machine Translation," Practical Experience of Machine Translation, pp. 61-71.

[Ross 82] Rossi F., 1982. "The Impact of Posteditors' Feedback on the Quality of MT," Practical Experi- ence of Machine Translation, pp. 113-117.

[Su 88] Su, K.-Y. and J.-S. Chang, 1988. "Semantic and Syntactic Aspects of Score Function," Proceedings of COLING-88, vol, 2, pp. 642-644, 12th International Conference on Computational Linguistics, Budapest, Hungary, August 22-27, 1988.

[Su 89] Su, K.-Y., J.-N., Wang, M.-H. Su, and J.-S. Chang, 1989. "A Sequential Truncation Parsing Algorithm Based on the Score Function," Proceedings of International Workshop on Parsing Technologies(IWPT 89), pp. 95-104, Pitsburgh, PA, USA.

[Su 90] Su, K.-Y., and J.-S. Chang, 1990. "Some Key Issues in Designing MT Systems," Machine Translation, vol. 5, no. 4, pp. 265-300, 1990.

[Su 91a] Su, K.-Y, and C.-H. Les, 1991. "Robustuess and Discrimination Oriented Speech Recognilion Using Weighting HMM and Subspace Projection Approaches," Proceedings of IEEE ICASSP-91. Toronto, Ontario, Canada.

[Su 91b] Su, K.-Y., J.-N. Wang, M.-H. Su, and J.-S. Chang, 1991. "GLR Parsing with Scoring," $M$. Tomita (ed.), Generalized $L R$ Parsing, Kluwer Academic Publishers.

[Wagn 74] Wagner, C.-K. and M.-J. Fischer, 1974. "The String-to-String Cornection Problem," Journal A.C.M., vol. 21, no. 1, pp. 168-173.

IWu 91 ] Wu., M.-W., J.-S. Chang and K.-Y. Su, 1991. "The Current Status of ArchTran: A Corpus-Based Statistics-Oriented English-Chinese Machine Translation System," Proceedings of the 1991 Workshop on Machine Translation, pp. 123-138, Nantou, Iraiwan, ROC, 24-26 June, 1991.

[Yu 91] Yu, S., 1991. "Automatic Evaluation of Output Quality for Machine Translation System," International Symposium on Multilingual Machine Translation, Beijing, China, pp. 57-58, 19-21 Aug. 1991. 